\title{
An Alternative Method for Determining Carbon Nanotube Band Energy
}

\author{
A. BAHARI* \\ Department of Physics, Faculty of Basic Science, University of Mazandaran, P.O. Box 47416, 467 Babolsar, Iran
}

(Received June 11, 2008; revised version October 5, 2008)

\begin{abstract}
Researchers calculated the electronic band energy of the carbon nanotube with some special form of crystalline potentials which yield to inaccurate equations. We thus modified the orthogonalized-plane-wave and tight binding methods. These methods are employed to introduce a matrix of carbon nanotube band energy.
\end{abstract}

PACS numbers: 04.62.+v, 98.80.Cq, 12.10.Dm

\section{Introduction}

Information technology is resulting from incredibly fast advances in electronics and computing during the last decades. Today, the electronics industry is producing metal-oxide-semiconductor field-effect transistors (MOSFETs) with critical dimensions of about $100 \mathrm{~nm}$, and projections anticipate devices with minimum future sizes of around $50 \mathrm{~nm}$ in the year 2009 [1-10]. However, some critical technological barriers and fundamental limitations to size reduction are threatening the use of orthogonalized-plane-wave (OPW) method for calculation of band energy. The main difficulty with current crystalline potentials resides quite simply in considering electrons of carbon atoms as independent particles.

One approach to overcoming these impending barriers involves finding on evaluating the potential of carbon nanotubes (CNTs) as the basis of a future nanoelectronics technology. Single-walled CNT (SWCNTs) are materials with unique properties. They have several millimeters in length and are strongly bonded covalent materials. Because of their extremely small diameter, the OPW method should be modified and completed with tight binding (TB) method due to the overlapping of wave function of electrons. The procedure is to augment the basis set of present method by including wave functions which are OPWs between nuclei of carbon atoms but represent modified Bloch waves near the nuclei. It means that by scaling the CNT dimension, the carbon atoms come close to each other and change the band energy. Thus, by using Ritz variational method [11], we have modified the band energy.

\section{Modifications of OPW method}

We consider Schrödinger equation as below

$$
\left[-\frac{\hbar^{2}}{2 m}+V(r)\right] \psi(r)=E \psi(r),
$$

* e-mail: a.bahari@umz.ac.ir where $V(r)$ is crystalline potential and $\psi(r)$ is the wave function of an electron in CNT structure. As with other variational methods, a trial wave function, $\psi(r)$, is tested on the system. In the Ritz method [12], the minimizing element in the $n$-th approximation is sought in the linear hull of the first $n$ coordinate elements. The Ritz ansatz function is a linear combination of $N$ orbitals. Based on linear combination of atomic orbital (LCAO) approximation, $\psi(r)$ is

$$
\psi(r)=\sum_{j} \phi_{j}(r) .
$$

The eigenvalues and eigenvectors can be found with finding a solution of the Ritz method. It is widely applied when solving eigenvalue problems, boundary value problems and OPW equations in general. The trial wave function will always give an expectation value larger than the ground energy (or at least, equal to it). It is known to be orthogonal to the ground state,

$$
\begin{aligned}
& H_{i j}=\left\langle\phi_{i}(r)|H| \phi_{j}(r)\right\rangle, \\
& P_{i j}=\left\langle\phi_{i}(r) \mid \phi_{j}(r)\right\rangle,
\end{aligned}
$$

where $j=1,2,3, \ldots, N$ and $N$ is the number of ground wave functions. Thus the associated equation is written by the following expression:

$$
\sum_{i}\left(H_{i j}, E P_{i j}\right) C_{i}=0 \text {. }
$$

The band energy $E(k)$ can be obtained by the Ritz method which is based on a variational determination of eigenvalues

$$
\operatorname{det}\left(H_{i j}, E P_{i j}\right)=0 \text {. }
$$

It is clear that there is an intense overlapping between electron wave functions of CNT when carbon atoms come close to each other. For this purpose we split ground OPW functions into two sections, a plane wave between atoms and $u(\boldsymbol{k}, \boldsymbol{r})$ (new wave functions) near to the atoms, i.e.:

$$
\phi_{i}(\boldsymbol{r})=A \mathrm{e}^{\mathrm{i} \boldsymbol{k}_{i} \cdot \boldsymbol{r}}-\sum_{s} C_{s u_{\mathrm{s}}}\left(\boldsymbol{k}_{i}, \boldsymbol{r}\right) .
$$


An approximation for the wave function of state can be obtained by finding the coefficients $C_{s u_{\mathrm{s}}}\left(\boldsymbol{k}_{i}\right)$ from the corresponding secular equation

$$
C_{s u_{\mathrm{s}}}\left(\boldsymbol{k}_{i}\right)=A \int u_{s}^{*}\left(\boldsymbol{k}_{i}, \boldsymbol{r}\right) \mathrm{e}^{\mathrm{i} \boldsymbol{k}_{i} \cdot \boldsymbol{r}} \mathrm{d}^{3} r .
$$

By inserting Eq. (6) into Eq. (5), we have

$$
\phi_{i}(\boldsymbol{r})=A\left[\mathrm{e}^{\mathrm{i} \boldsymbol{k}_{i} \cdot \boldsymbol{r}}-\sum_{s}\left\langle u_{s}\left(\boldsymbol{k}_{i}, \boldsymbol{r}\right) \mathrm{e}^{\mathrm{i} \boldsymbol{k}_{i} \cdot \boldsymbol{r}}\right\rangle u_{s}\left(\boldsymbol{k}_{i}, \boldsymbol{r}\right)\right] .
$$

Now, the matrix array of OPW results by

$$
H_{i j}-E P_{i j}=\left\langle\phi_{i}(r)|H| \phi_{j}(r)\right\rangle-E\left\langle\phi_{i}(r) \mid \phi_{j}(r)\right\rangle .
$$

By doing series of calculations, the matrix arrays are

$$
\begin{gathered}
M_{i j}=\left(\boldsymbol{k}_{i} \cdot \boldsymbol{k}_{j}-E\right) \delta_{i j}+V_{i j} \\
+\sum_{s}\left[E-E_{s}(\boldsymbol{k})\right] C_{s i} C_{s j},
\end{gathered}
$$

where $V_{i j}$ are the coefficients of the Fourier expansion of a potential function.

\section{An alternative method of electronic band structure}

As one can see in Eq. (8), $V_{i j}$ indicate that there are no interactions between electrons of carbon atoms, i.e., each electron is imagined as a nearly free electron. Obviously, the above assumptions cannot explain the electron's behavior when carbon atoms come together like $d$-layer electrons.

In this case we deform new wave function based on orthogonalized-plane-wave and tight binding methods [13]. Therefore, $u\left(\boldsymbol{k}_{i}, \boldsymbol{r}\right)$ should be modified based on TB and OPW methods. For $N$ unit cells in CNTs, $u\left(\boldsymbol{k}_{i}, \boldsymbol{r}\right)$ can be written by the following expression:

$$
u\left(\boldsymbol{k}_{i}, \boldsymbol{r}\right)=\frac{1}{\sqrt{N}} \sum_{n=1} \mathrm{e}^{\mathrm{i} \boldsymbol{k}_{i} \cdot \boldsymbol{R}_{n}} \psi_{n l m}\left(\boldsymbol{r}-\boldsymbol{R}_{n}\right),
$$

where $\boldsymbol{R}_{n}$ is the distance between two nearest neighbor carbon atoms. It yields new orthogonalized coefficients. We consider a correction term as $L_{i j}$,

$$
M_{i j}=\left(\boldsymbol{k}_{i} \cdot \boldsymbol{k}_{j}-E\right) \delta_{i j}+L_{i j} \text {. }
$$

By using separable variables method, the atomic wave functions, $\psi_{n l m}(\boldsymbol{r})$, split into a set of radial $R_{n l}(\boldsymbol{r})$, azimuth angle part $\phi_{m}(\varphi)$ and associated Legendre equation $P_{l m}(x)$, with $x=\cos \theta$.

We show that $E_{s}(\boldsymbol{k}) \simeq E_{n l}$ as a special band energy of SWCNT electrons in which

$$
\begin{aligned}
& L_{i j}=V_{i j}+4 \pi A \sum_{n, l}(2 l+1) P_{l}\left(\boldsymbol{k}_{i}, \boldsymbol{k}_{j}\right)\left(E-E_{n l}\right) \\
& \quad \times a_{n l}^{*}\left(\boldsymbol{k}_{i}\right) a_{n l}\left(\boldsymbol{k}_{j}\right),
\end{aligned}
$$

where $a_{n l}$ can be determined by using the spherical Bessel functions, $j_{l}$, as below

$$
a_{n l}\left(\boldsymbol{k}_{i}\right)=\int_{0}^{\infty} j_{l}\left(\boldsymbol{k}^{\prime}\right) \boldsymbol{r} R_{n l}(\boldsymbol{r}) r^{2} \mathrm{~d}^{3} r
$$

From Refs. [11, 12, 14-18], $\psi(\boldsymbol{r})$ can be written as below

$$
\psi(\boldsymbol{r})=\sum_{i} C_{i}\left(\boldsymbol{k}_{i}\right) \phi\left(\boldsymbol{k}^{\prime}, \boldsymbol{r}\right)+\sum_{s} a_{s}(\boldsymbol{k}) u_{s}(\boldsymbol{k}, \boldsymbol{r}),
$$

where $a_{s}, c_{i}$ coefficients are atom coordinate elements in the Ritz variational method [12]. We separate Eq. (13) into two parts, self atom and the nearest atoms of concern atom, i.e.,

$$
\begin{aligned}
& u_{s}(\boldsymbol{k}, \boldsymbol{r})=\sum_{n} \mathrm{e}^{\mathrm{i} \boldsymbol{k} \cdot \boldsymbol{R}_{n}} \phi_{s}\left(\boldsymbol{r}-\boldsymbol{R}_{n}\right) \\
& -\sum_{s^{\prime}} b_{s s^{\prime}} \psi_{s^{\prime}}(\boldsymbol{r}),
\end{aligned}
$$

where

$$
b_{s s^{\prime}}=\int_{0}^{\infty} \psi_{s^{\prime}}(\boldsymbol{r}) u_{s}(\boldsymbol{k}, \boldsymbol{r}) \mathrm{d}^{3} r .
$$

Therefore the electronic band energy is determined by the following equation:

$$
\operatorname{det}|H-E P|=0 \text {, }
$$

where

$$
\begin{gathered}
\left(\begin{array}{cc}
H_{i j}^{\mathrm{OPW}} & H_{i s} \\
H_{i s}^{\dagger} & H_{s s}
\end{array}\right), \\
\left(\begin{array}{cc}
P_{i j}^{\mathrm{OPW}} & P_{i s} \\
P_{i s}^{\dagger} & P_{s s}
\end{array}\right) .
\end{gathered}
$$

Finally, the overlapping wave functions of SWCNTs is demonstrated by $H$ and $P$ matrices,

$$
\begin{aligned}
& H_{i s}=\int_{0}^{\infty} \phi_{i}(\boldsymbol{r}) H u_{s}(\boldsymbol{k}, \boldsymbol{r}) \mathrm{d}^{3} r-\sum_{q} E_{q} a_{q} b_{s q}, \\
& H_{s s^{\prime}}=\int_{0}^{\infty} \phi_{i}(\boldsymbol{r}) u_{s}(\boldsymbol{k}, \boldsymbol{r}) H u_{s^{\prime}}(\boldsymbol{k}, \boldsymbol{r}) \mathrm{d}^{3} r \\
& \quad-\sum_{q} E_{q} b_{s q} b_{s^{\prime} q},
\end{aligned}
$$

and

$$
\begin{aligned}
P_{i s} & =\int_{0}^{\infty} \phi_{i}(\boldsymbol{r}) u_{s}(\boldsymbol{k}, \boldsymbol{r}) \mathrm{d}^{3} r-\sum_{q} C_{q} b_{s q}, \\
P_{s s^{\prime}} & =\int_{0}^{\infty} \phi_{i}(\boldsymbol{r}) u_{s}(\boldsymbol{k}, \boldsymbol{r}) u_{s^{\prime}}(\boldsymbol{k}, \boldsymbol{r}) \mathrm{d}^{3} r \\
& -\sum_{q} b_{s q} b_{s^{\prime} q},
\end{aligned}
$$

where

$$
b_{s q}=\sum_{n} \mathrm{e}^{\mathrm{i} \boldsymbol{k} \cdot \boldsymbol{R}_{n}} \int_{0}^{\infty} \psi_{q}(\boldsymbol{r}) \phi_{s}\left(\boldsymbol{r}-\boldsymbol{R}_{n}\right) \mathrm{d}^{3} r .
$$

\section{Conclusion}

We have demonstrated the series of calculations based on the Ritz variational, OPW and TB methods. We have found a reliable matrix which can describe the CNT electron behavior, correctly. In this method, there is no limitation on crystalline potential of CNT structure, so it can be suggested for evaluating the electronic band energy of SWCNTs. 


\section{Acknowledgments}

This work is supported with a grant from the University of Mazandaran.

\section{References}

[1] Y.J. Lee, J. Cryst. Growth 266, 568 (2004).

[2] A. Bahari, U. Robenhagen, P. Morgen, Z.S. Li, Phys. Rev. B 72, 205323 (2005).

[3] A. Bahari, P. Morgen, Z.S. Li, Surf. Sci. 600, 2966 (2006).

[4] A. Bahari, P. Morgen, K. Pedersen, Z.S. Li, J. Vac. Technol. B 24, 2119 (2006).

[5] T. Bieniek, A. Wojtkiewicz, L. Lukasiak, R.B. Beck, Biochem. Soc. Trans. 29, 702 (2001).

[6] P. Morgen, A. Bahari, U. Robenhagen, J. Anderson, K. Pedersen, M.G. Rao, Z.S. Li, J. Vac. Technol. A 23, 201 (2005).

[7] A. Bahari, P. Morgen, Z.S. Li, Surf. Sci. 602, 2315 (2008).
[8] A. Bahari, P. Morgen, K. Pedersen, Z.S. Li, J. Phys. Conf. Series 86, 012019 (2007).

[9] P.R. Giri, Int. J. Theor. Phys. 47, 1776 (2008).

[10] B. Chen, X. Shen, L. Sun, R. HanGiri, Int. J. Theor. Phys. 46, 199 (2007).

[11] J. Appenzeller, E. Joselevich, W. Hoenlein, Nanoelectronics and Information Technology, Wiley-VCH, Weinheim 2003.

[12] W. Ritz, J. Reine, J. Appl. Mech. 135, 1 (1909).

[13] A. Bahari, M. Amiri, Acta Phys. Pol. A 115, (2009).

[14] K. Schwarz, Phys. Rev. B 5, 24 (1972).

[15] J. Callaway, Phys. Rev. B 99, 500 (1955).

[16] R.A. Deegan, W.D. Twose, Phys. Rev. 164, 993 (1967).

[17] H. Salehi, Computational Methods in Solid State Physics, Shahid Chamran University Press, Iran 2007.

[18] J. Zhu, X.M. Wang, S.G. Louie, Phys. Rev. B 45, 8887 (1992). 